\title{
La atención del parto en Chiapas, México: ¿dónde y quién los atiende?
}

Héctor Javier Sánchez-Pérez, C.D., M. en I.S.S., M.Sc., ${ }^{(1,2)}$ Héctor 0 choa-Díaz López, M.C., M. Sc., Ph. D., ${ }^{(1)}$ Albert Navarro i Giné, D. en Estadística, ${ }^{(2)}$ Miguel Martín-Mateo, Ph. D. ${ }^{(2)}$

\section{Sánchez-Pérez HJ, Ochoa-Díaz López H, Navarro i Giné A, Martín-Mateo $M$. La atención del parto en Chiapas, México: ¿dónde y quién los atiende? Salud Publica Mex 1998;40:494-502.}

\section{Resumen}

Objetivo. Identificar el sitio y el agente de atención del parto; analizar el agente de atención del parto según la atención prenatal (AP) y factores sociodemográficos; identificar grupos con menor y mayor probabilidad de recibir atención por parte del personal de los servicios de salud; identificar los motivos de no atenderse en la unidad de salud más cercana al domicilio. Material y métodos Se analizan datos sobre la atención del parto en 297 mujeres de la Región Fraylesca (Chiapas), usando modelos logit para la identificación de grupos. Resultados El 32\% de los partos ocurrieron en instituciones de salud, y $60 \%$, en el hogar (mayo ritariamente en condiciones higiénico-sanitarias desfavorables). Sólo $10 \%$ del grupo de mujeres con menos de cinco consultas de AP, una escolaridad menor al tercero de primaria y un jefe de hogar dedicado a labores agropecuarias, fue atendido por personal de salud. Conclusiones Es necesario mejorar la cobertura, la aceptabilidad y la calidad de los servicios de salud e instrumentar un programa dirigido a incrementar el número de partos en condiciones higiénico-sanitarias adecuadas.

Palabras clave: atención del parto; atención prenatal; parteras tradicionales; calidad de la atención de la salud; accesibilidad a los servicios de salud; México

\author{
Sánchez-Pérez HJ, Ochoa-Díaz López H, \\ Navarro i Giné A, Martín-Mateo M. \\ Childbirth in Chiapas, Mexico: \\ Who provides delivery care and where? \\ Salud Publica Mex 1998;40:494-502.
}

\begin{abstract}
A bstract
Objective. To identify the place and provider of delivery care; to analyse the relationship between the type of delivery care provider and prenatal care and sociodemographic factors; to identify groups with greater and lesser probability of receiving attention at health centers and to identify the reasons for not attending the health center nearest to the household. Material and methods Data on the delivery care of 297 women of La Fraylesca Region, C hiapas, were gathered using multivariate logit models to identify groups. Results From the total, $32 \%$ of childbirths occurred at health centers and $60 \%$ at home (mostly with poor sanitary conditions). 0 nly 10\% of women with less than 5 prenatal visits, school level under 3 years and whose household head was a peasant were attended by health care personnel. Conclusions The accessibility and quality of health centers must be improved, and a programme aimed at increasing the number of deliveries that are attended by trained health care personnel should be implemented.
\end{abstract}

Key words: delivery care; prenatal care; midwives, practical;quality of health care; health ser vices accessibility; M exico

(1) División de Población y Salud, El Colegio de la Frontera Sur (EC O SUR), San Cristóbal de las Casas, Chiapas, México.

(2) Laboratorio de Bioestadística y Epidemiología de la Facultad de Medicina, U niversidad Autónoma de Barcelona (UAB), Bellaterra, Barcelona, España.

Fecha de recibido: 12 de febrero de 1998 • Fecha de aprobado: 24 de septiembre de 1998 Solicitud de sobretiros: Dr. Héctor Javier Sánchez Pérez. El Colegio de la Frontera Sur. Carretera Panamericana y Periférico Sur s/n, 29290 San Cristóbal de las Casas, Chiapas, México. Correo electrónico: hsanchez@ sclc.ecosur.mx 
M éxico ocupa, dentro del continente americano, un nivel "intermedio" en cuanto a mortalidad materna se refiere. En 1990 la tasa estimada fue de 110 muertes maternas por cada 100000 nacidos vivos; si bien está muy por debajo de las notificadas para Bolivia y Haití (650 y 1 000, respectivamente), dicha tasa aún se ubica muy por arriba de la señalada para Canadá y Estados Unidos de América (6 y 12, respectivamente). ${ }^{1}$

Entre las principales alternativas para disminuir la mortalidad y la morbilidad materna -y perinatalse encuentra la de brindar una atención adecuada y oportuna a la mujer durante el embarazo, el parto y el puerperio. ${ }^{1,2}$ Hoy en día se considera que la mayoría de las muertes maternas puede evitarse con una adecuada y pronta atención prenatal y obstétrica., En cuanto a la morbilidad, según estimaciones del Fondo de las Naciones Unidas para la Infancia, ${ }^{1}$ por cada mujer que muere durante el parto, aproximadamente otras 30 sufren lesiones y contraen infecciones, muchas de las cuales no son atendidas ni tratadas; ello a su vez provoca discapacidades y molestias que pueden perdurar en la mujer, incluso, para toda su vida.

En México, Chiapas es la segunda entidad con el mayor crecimiento poblacional anual ${ }^{5} \mathrm{y}$ con la mayor tasa global de fecundidad. ${ }^{6}$ Sin embargo, también es la entidad con la marginación socioeconómica más alta del país y constituye una de las más desfavorecidas respecto al monto de recursos destinados a la salud por habitante. Datos oficiales de $1992^{7}$ ubicaban a Chiapas como el estado con la menor proporción de partos atendidos por médico en hospital o clínica ( $14 \%$ vs 69\% en el ámbito nacional), con el número más alto de partos atendidos por partera o enfermera en domicilio $(63 \%$ vs $26 \%)$ y con la de mayor proporción de nacimientos consignados en los registros oficiales sin especificar el agente y el sitio de atención (23\% vs 4\%). Para 1995, datos de la Encuesta Nacional de Planificación Familiar $1995^{6}$ ubicaban a Chiapas como el segundo estado del país con el menor porcentaje de partos atendidos en hospitales públicos $(42 \%)$, precedido sólo por el estado de Guerrero (41\%), en tanto que en el ámbito nacional dicho indicador fue de $66 \%$; la entidad en mejor situación fue el Distrito Federal, con prácticamente $100 \%$.

Diversos estudios han puesto de manifiesto que, tanto los niveles de fecundidad, como el número de partos que tienen lugar sin atención alguna, no se distribuyen de manera uniforme entre la población. En cuanto a la fecundidad, se ha documentado extensamente su fuerte asociación con los niveles de marginación social (a mayor marginación, mayores niveles de fecundidad). ${ }^{8}$ Respecto a la atención del parto, también existe una relación en el mismo sentido. Así, se ha señalado que entre las mujeres con escolaridad de hasta tercero de primaria, provenientes de comunidades indígenas y cuya casa tiene piso de tierra, sólo uno de cada nueve partos tendrá lugar en alguna institución de salud, ${ }^{9}$ en tanto que una alta proporción de los partos atendidos en casa de la mujer o de las parteras tiene lugar en condiciones de higiene inadecuadas. ${ }^{4}$

Estos datos revelan que, en términos generales, los grupos poblacionales con mayor número de hijos y con mayores desventajas socioeconómicas son los que reciben menor atención materna, entre otro tipo de servicios. Debido a tales consideraciones, a fines de 1994 se llevó a cabo un proyecto de investigación encaminado a analizar el bienestar social de la población urbana y rural de la Región Fraylesca de Chiapas y su relación con las necesidades de salud no satisfechas de la población, así como el uso, la cobertura y la equidad en los sistemas de atención a la salud, incluidos el uso de servicios de atención prenatal y del parto. ${ }^{*}$ En un artículo anterior se presentaron los resultados relativos a la atención prenatal. ${ }^{10}$ En este trabajo se exponen los resultados obtenidos en lo referente al análisis de la atención del parto con los siguientes objetivos planteados:

1. Identificar el sitio y el agente de atención del parto.

2. Analizar si existe o no relación entre el agente de atención del parto y: a) el número de consultas recibidas durante la atención prenatal; b) el agente de salud que brindó la atención prenatal, y c) diversos factores demográficos, socioeconómicos y de servicios de salud.

3. Identificar motivos de la no atención del parto en la unidad de salud más cercana.

4. Identificar grupos de mujeres con menor y mayor probabilidad de recibir atención del parto por parte de médicos o enfermeras de los servicios de salud (públicos o privados).

\section{Material y métodos}

\section{Población estudiada}

La Región Fraylesca de Chiapas es una zona de alta ruralidad, con poco más de 183000 habitantes ${ }^{11}$ distribuidos en 958 localidades, de las que sólo cinco rebasan

* Ochoa-Díaz H, Sánchez-Pérez HJ. Proyecto: Bienestar social, necesidades y servicios de salud en la Región Fraylesca de Chiapas. San Cristóbal de las Casas: El Colegio de la Frontera Sur, 1994. Documento no publicado. 
los 2500 habitantes y sólo una supera los 10000 pobladores. Está conformada por cuatro de los 111 municipios de Chiapas (Villaflores, Jaltenango -llamado también Angel Albino Corzo-, Villacorzo y la Concordia) y, si bien es una zona con poca presencia de grupos indígenas, tiene una elevada proporción de grupos campesinos en condiciones de pobreza extrema. ${ }^{11}$ En términos socioeconómicos esta región está considerada de "alta marginación" -con excepción del municipio de Villaflores, clasificado de "media marginación" ${ }^{\prime \prime},{ }^{12}$ y prácticamente hasta ahora, no se cuenta con estudios que revelen las condiciones y necesidades de salud no satisfechas de su población.

\section{Diseño y tamaño de la muestra}

Se eligió una muestra aleatoria ( $\mathrm{n}=1100$ viviendas), estratificada (área urbana / rural) y por conglomerados, de los dos municipios que guardan condiciones socioeconómicas extremas en la región (Villaflores, el de mejor situación, y Jaltenango, el de peor situación); posteriormente se realizó una encuesta donde se recopiló información sociodemográfica, de salud y de uso de servicios de salud, entre los que se incluyó la atención prenatal y del parto. El tamaño muestral para los dos municipios estudiados se determinó tomando en consideración una precisión de 0.15 y un nivel de confianza de $90 \%$ en los estimadores buscados para el proyecto en su conjunto.

El seguimiento sobre la atención prenatal y del parto (sitio y agente de atención) se efectuó, de manera directa, en todas aquellas mujeres de 15-49 años de edad que finalizaron su embarazo en los dos años previos al estudio $(n=297)$.

Los indicadores demográficos, socioeconómicos y de servicios de salud utilizados para analizar los factores que inciden en el tipo de agente de atención del parto, así como en la identificación de mujeres con menor y mayor probabilidad de ser atendidas durante el parto por personal de los servicios de salud, fueron los siguientes: a) demográficos: edad, edad del primer embarazo; b) socioeconómicos: escolaridad y ocupación de la madre y del jefe del hogar, estrato de la localidad de residencia, seguridad social, frecuencia de ingesta de carne -como indicador de ingreso-, condiciones de la vivienda (tipo de piso, número de cuartos, tipo de cocina, disponibilidad de luz, agua, refrigerador, forma de eliminación de excretas); c) de servicios de salud: distancia a la unidad de salud más cercana al domicilio, número de consultas y agente de salud que brindó la atención prenatal, y sitio de atención del parto.

\section{A nálisis estadístico}

Para el análisis crudo entre la variable respuesta (agente de atención del parto) y los factores estudiados, se realizaron pruebas de independencia con el estadístico $\chi^{2}$, con un nivel de significación $\alpha=0.05$; en el caso de variables cuantitativas, para comprobar diferencias entre muestras, se utilizó el test no paramétrico de Kruskal-Wallis, debido al reducido tamaño poblacional de uno de los grupos (mujeres que no recibieron atención sanitaria alguna durante el parto).

Para la identificación de grupos de mujeres con menor y mayor probalidad de recibir atención del parto por personal de salud (médicos o enfermeras de servicios públicos o privados), se utilizó un modelo logit construido ad hoc a partir de las variables analizadas que mostraron asociación estadísticamente significativa con la variable respuesta. El ajuste del modelo logit utilizado se realizó con las mismas técnicas de modelación utilizadas en el estudio relativo a la atención prenatal en esta misma población; ;0,13,14 por este motivo, la variable respuesta, que originalmente fue categorizada en tres grupos (mujeres que recibieron atención por personal de los servicios de salud, las atendidas por la partera y mujeres sin atención alguna), fue dicotomizada uniendo las dos últimas categorías. De esta manera, la variable estudiada mediante el modelo logit fue si el parto había sido o no atendido por personal formal de los servicios de salud.

Las variables explicativas utilizadas en el modelo fueron: número de consultas recibidas durante la atención prenatal, 1=0-4, 2= 5 y más; ${ }_{1}^{15}$ ocupación del jefe del hogar, $1=$ agropecuarias, $2=$ no agropecuarias; escolaridad de la madre, $1=\leq 30$. de primaria, $2=>30$. de primaria, y edad del primer embarazo de la madre, $1=$ $\leq$ de 15 años, $2=>$ de 15 .

Asimismo, con el fin de hacer más comprensibles los resultados obtenidos en términos epidemiológicos, una vez que se aplicaron al modelo los criterios de ajuste y la mayor parsimonia posible, ${ }^{13,14}$ se deshizo la transformación logit para obtener las razones de probabilidad y facilitar la interpretación en el análisis en términos de razones de prevalencia. Para todos estos análisis de datos se utilizaron los paquetes estadísticos SPSS, Stat Xact, versión 2.11, y Glim-4. ${ }^{16}$ En todos los contrastes de independencia efectuados, se utilizó un nivel de significación estadística de $p<0.05$.

\section{Resultados}

De las 1100 viviendas seleccionadas para la muestra, se recopiló información de 1046 (tasa de respuesta de 
95\%). El número de población censada fue de 5 548, entre las que se encontraron 297 mujeres con embarazo concluido en el transcurso de los dos años previos al estudio, de las que sólo una se negó a responder la sección correspondiente a la atención prenatal y del parto.

En términos generales, el grupo de mujeres estudiadas $(n=296)$ es una población joven (edad promedio de 26 años) con altos niveles de pobreza, bajo nivel educativo, en su mayoría dedicada a labores dentro del hogar, sin seguridad social y con condiciones deficientes de la vivienda. En cuanto a la atención prenatal recibida, la mediana del número de consultas fue de cinco.

\section{Sitio y agente de atención del parto}

Sitio de atención. De los 296 partos analizados, 95 (32\%) tuvieron lugar en alguna institución de salud; $15(5 \%)$, en casa de la partera; 178 (60\%), en casa de la mujer; siete, en casa de la mamá de la mujer o de la suegra, y uno, en un vehículo. De los 95 atendidos en instituciones de salud, 69 se llevaron a cabo en servicios de salud "abiertos"; nueve en servicios "cerrados", y 17 en servicios privados.

Sólo 8\% ( $\mathrm{n}=24)$ de los 296 partos analizados tuvieron lugar en la unidad de salud más cercana al domicilio de las mujeres estudiadas. Los motivos que señalaron las mujeres que no se atendieron en dicho centro fueron, principalmente, la percepción de mala atención y la falta de confianza en dichos centros (cuadro I).

Por otra parte, las condiciones de la vivienda en que tuvieron lugar los partos de 178 mujeres estudiadas fueron las siguientes: $141(79 \%)$ tenían un solo cuarto para dormir. La mediana observada del número de personas por cuarto para dormir fue de seis, mientras que el porcentaje de viviendas con 1-3 personas por cuarto fue de $13 \%$; con 4-6 personas, de $51 \%$, y con siete o más, de $37 \%$. Asimismo, $153(86 \%)$ de las viviendas contaban con paredes de tierra o adobe; 87 (49\%), con piso de tierra; 69 (39\%) no tenían agua entubada; 135 (76\%) no disponían de baño con excusado o retrete; 32 (18\%) carecían de electricidad, y $148(83 \%)$, de refrigerador. Por último, $142(80 \%)$ viviendas tenían cocina de leña. Tal como puede apreciarse, la mayoría de las viviendas presentaron condiciones higiénico-sanitarias inadecuadas para la atención del parto.

Agente que atendio el parto. La partera asistió en 169 (57\%) partos; 95 (32\%) fueron atendidos por médicos; $14(5 \%)$, por una enfermera, y $18(6 \%)$, por un familiar $(n=12)$ o no recibieron atención alguna $(n=6)$. En el cuadro II se muestra el tipo de agente que atendió el parto según el sitio donde tuvo lugar. Tal como se esperaba, los 18 partos que no recibieron atención sanitaria alguna se ubicaron dentro del grupo de partos ocurridos en casa de la mujer $(n=178)$ o en casa de su mamá o suegra $(\mathrm{n}=7)$.

\section{Cuadro I}

Razones por las que las mujeres no se atendieron Del PARTO EN LA UNIDAd de SALUd MÁs CERCANA A SU CASA. Región Fraylesca, Chiapas, México, 1994

Razón de la no atención en el centro de salud más cercano a su casa

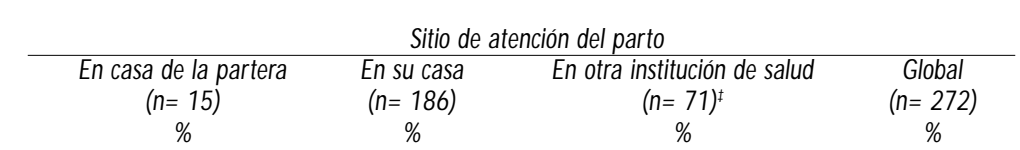

Percepción de mala atención y falta de confianza*

\begin{tabular}{|c|c|c|c|c|}
\hline Percepción de mala atención y falta de confianza* & 54 & 35 & 53 & 40 \\
\hline No fue necesario & 26 & 28 & 8 & 23 \\
\hline Está muy lejos & 13 & 13 & 11 & 13 \\
\hline Falta de dinero & - & 15 & 7 & 12 \\
\hline No le gusta que la vean los médicos, prefiere a la partera & 7 & 6 & - & 5 \\
\hline Del centro de salud la mandaron al hospital & - & - & 17 & 4 \\
\hline 0 tros (tiene seguro, no había quién cuidara a sus hijos) & - & 3 & 4 & 3 \\
\hline
\end{tabular}

* El tipo de respuestas agrupadas bajo esta categoría fueron: "ahí atienden mal”, "ahí no curan", "no le tengo confianza”, "no tienen equipos para atender ahí" y "nunca está el doctor"

₹ Se incluyó a cualquier servicio de salud que no fuera la unidad de salud más cercana al domicilio de la mujer (bien sea de la Secretaría de Salud o del Programa IMSS-Solidaridad) 


\section{Cuadro II

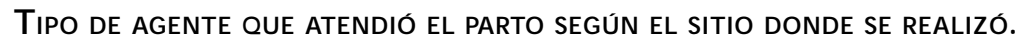 Region fraylesca, Chiapas, Mexico, 1994}

Agente que atendió el parto

Sola o familiar

Partera 82.8

Enfermera

Total

100.0
$(n=186)$

$\begin{array}{cc}\text { Casa de la mujer, } & \text { Casa de la } \\ \text { madre o suegra* } & \text { partera } \\ \% & \%\end{array}$

Sitio donde ocurrió el parto

$\begin{array}{cc}\text { Servicios de salud abiertos } & \text { Otros servicios } \\ \text { (primer nivel de atención) } & \text { de salud } \\ \% & \%\end{array}$

* Incluye 178 casos o curridos en casa de las mujeres; 7, en casa de las madres o suegras de las mujeres, y un caso atendido en un vehículo por una partera

₹ Incluye servicios de salud abiertos de segundo nivel de atención $(n=43)$, servicios cerrados $(n=9)$ y privados $(n=17)$

Agente que atendio el parto según el número de consultas recibidas durante la atención prenatal. Se encontró una mayor proporción de mujeres sin atención alguna durante el parto entre las que recibieron menos de cinco consultas de atención prenatal (AP), que entre aquellas que recibieron cinco y más $(p<0.05)$. De las 124 que recibieron 0-4 consultas, $65 \%$ fueron atendidas en el parto por la partera; $23 \%$, por personal médico, y $11 \%(\mathrm{n}=14)$ no recibieron atención por personal sanitario. De las 172 mujeres que recibieron cinco o más consultas de $\mathrm{AP}, 51 \%$ fueron asistidas por la partera; $47 \%$, por personal de salud, y sólo $2 \%(\mathrm{n}=4)$ no recibió atención sanitaria durante el parto. La razón de momios cruda de haber sido atendida durante el parto por personal de salud en las mujeres que recibieron cinco o más consultas de $\mathrm{AP}$, respecto a las que tuvieron de 0 a 4 consultas, fue de 2.85 (IC95\% 1.71-4.76).

Finalmente, y en concordancia con lo anterior, cabe destacar que la mediana de consultas de AP entre las mujeres que no recibieron atención sanitaria en el parto fue de 1.5; la de las mujeres que fueron asistidas por la partera, de cinco, y la de aquellas que contaron con la asistencia de personal de los servicios de salud, de siete $(p<0.05)$.

Agente que atendio el parto según agente de salud que proporcionó la atención prenatal. Entre las mujeres que no tuvieron atención prenatal alguna, es decir, cero consultas $(n=23), 50 \%$ fueron atendidas, durante el parto, por la partera; $18 \%$, por personal de servicios de salud abiertos, y $32 \%$ no recibió atención sanitaria. Por su parte, el agente que atendió el parto según el agente que brindó la atención prenatal se muestra en el cuadro III.
Agente de salud que atendio el parto según las características demográficas y socioeconómicas de las mujeres estudiadas. De las variables demográficas analizadas, se encontró que, respecto a la edad de las mujeres, al categorizar esta variable en dos grupos (uno conformado por las mujeres de hasta 20 años de edad y por las de 35 a 44 años -"grupo de riesgo"-, y otro, por las de 21 a 34 años), no hubo diferencias estadísticamente significativas. Sin embargo, el grupo de mujeres que no recibió atención durante el parto fue el que mostró la mediana de edad más grande: 29 años vs 25 y 26 años del grupo atendido por parteras y servicios de salud, respectivamente $(p<0.05)$.

Respecto a la edad del primer embarazo, se encontró que las mujeres que declararon haber tenido su primer embarazo a los 20 o más años de edad, contaron con mayor atención del parto por parte del personal de salud que aquellas que tuvieron su primer embarazo antes de los 20 años de edad (cuadro IV). Asimismo, no se apreciaron diferencias respecto a la condición de si hablaban o no alguna lengua indígena.

En cuanto a las variables socioeconómicas analizadas, prácticamente en todas se observaron diferencias estadísticamente significativas en relación con la variable respuesta (cuadro IV). Sin embargo, debe destacarse que las mujeres que no recibieron atención sanitaria durante el parto $(n=18)$ constituyeron el grupo en peores condiciones de pobreza y marginación. No obstante, con excepción de las variables piso de tierra, tipo de cocina en la vivienda y frecuencia de ingesta de carne-variables en las que también mostraron las peores condiciones-, este grupo fue muy similar en términos socioeconómicos al grupo de mujeres que fueron atendidas por las parteras $(n=169)$. De esta ma- 


\section{Cuadro III}

\section{Agente que atendió el parto según el agente que proporcionó la atención prenatal. Región Fraylesca, Chiapas, México, 1994}

\begin{tabular}{|c|c|c|c|c|c|}
\hline \multirow[b]{2}{*}{ Agente que atendió el parto } & \multicolumn{5}{|c|}{ Agente de atención prenatal } \\
\hline & $\begin{array}{c}\text { Servicios abiertos } \\
\%\end{array}$ & $\begin{array}{c}\text { Servicios cerrados } \\
\%\end{array}$ & $\begin{array}{c}\text { Servicios privados } \\
\%\end{array}$ & $\begin{array}{l}\text { Parteras } \\
\%\end{array}$ & Sin atención sanitaria \\
\hline Servicios abiertos* & 48.6 & 8.3 & 28.6 & 5.2 & 18.0 \\
\hline Servicios cerrados (médicos) & - & 58.3 & 6.1 & 2.1 & - \\
\hline Servicios privados (médicos) & 1.8 & 8.3 & 24.5 & 3.1 & - \\
\hline Partera & 48.6 & 25 & 38.8 & 82.3 & 50.0 \\
\hline Sin atención sanitaria & 0.9 & - & 2.0 & 7.3 & 32.0 \\
\hline Total & 100.0 & 100.0 & 100.0 & 100.0 & 100.0 \\
\hline & $(n=111)$ & $(n=12)$ & $(n=49)$ & $(n=96)$ & $(n=23)$ \\
\hline
\end{tabular}

*Incluye partos atendidos por médicos y enfermeras de los servicios de salud abiertos

\begin{tabular}{|c|c|c|c|c|}
\hline \multicolumn{5}{|c|}{ 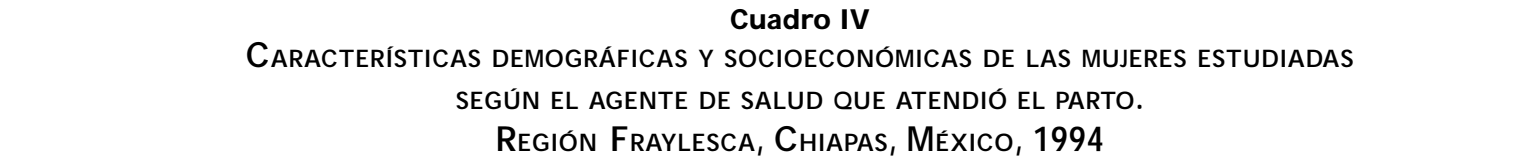 } \\
\hline Indicador & & $\begin{array}{c}\text { Servicios de salud } \\
\quad(n=109) \\
\text { RM (IC95\%) }\end{array}$ & $\begin{array}{c}\text { te que atendió el } p \\
\text { Partera } \\
(n=169) \\
\text { RM (IC95\%) }\end{array}$ & $\begin{array}{c}\text { Nadie/familiar } \\
(n=18) \\
\text { RM (IC95\%) }\end{array}$ \\
\hline \multicolumn{5}{|l|}{ Demográficos } \\
\hline Edad del primer embarazo (años) & $\geq 20$ & $2.71(1.62-4.56)$ & $0.42(0.25-0.69)$ & $0.67(0.21-2.08)$ \\
\hline & $<20^{*}$ & & & \\
\hline \multicolumn{5}{|l|}{ Socioeconómicos } \\
\hline \multirow[t]{2}{*}{ Escolaridad: } & $>30$. de primaria & $3.53(2.15-5.80)$ & $0.40(0.25-0.65)$ & $0.18(0.04-0.80)$ \\
\hline & $\leq 30$. de primaria* & & & \\
\hline \multirow[t]{2}{*}{ Analfabetismo: } & no & $3.27(1.80-5.93)$ & $0.36(0.21-0.62)$ & $0.83(0.30-2.28)$ \\
\hline & sí* & & & \\
\hline \multirow[t]{2}{*}{0 cupación, hogar: } & no & $3.46(1.34-8.97)$ & $0.38(0.15-0.99)$ & $\neq$ \\
\hline & sí* & & & \\
\hline \multirow[t]{2}{*}{0 cupación jefe del hogar: } & no agropecuaria & $3.46(2.07-5.80)$ & $0.41(0.24-0.67)$ & $0.12(0.02-0.94)$ \\
\hline & agropecuaria* & & & \\
\hline \multirow[t]{2}{*}{ Ingesta de carne (por semana): } & $>1 \mathrm{vez}$ & $3.28(1.96-5.51)$ & $0.58(0.36-0.92)$ & $0.04(0.01-0.30)$ \\
\hline & $\leq$ de 1 vez* & & & \\
\hline \multirow[t]{2}{*}{ Localidad de residencia: } & urbana & $4.59(2.75-7.64)$ & $0.31(0.19-0.50)$ & $0.21(0.05-0.93)$ \\
\hline & rural* & & & \\
\hline Con seguridad social: & sí & $5.34(2.27-12.5)$ & $0.25(0.11-0.59)$ & $\neq$ \\
\hline
\end{tabular}

\section{Características de la vivienda}

Tipo de piso:

\begin{tabular}{lccc} 
con recubrimiento & $2.56(1.53-4.27)$ & $0.63(0.39-1.01)$ & $0.17(0.06-0.54)$ \\
\hline sin recubrimiento (tierra)* & & & \\
\hline con gas & $5.96(3.54-10.0)$ & $0.28(0.17-0.46)$ & \\
\hline con leña* & & & \\
sí & $5.83(2.01-16.93)$ & $0.43(0.20-0.92)$ & $0.26(0.09-0.74)$ \\
\hline no* & & & \\
sí & $3.28(1.92-5.61)$ & $0.41(0.24-0.70)$ & $0.15(0.02-1.17)$ \\
\hline no* & &
\end{tabular}

* Categorías de referencia

₹ Todos los casos se ubicaron en la casilla de referencia 
nera, puede señalarse que estos dos grupos presentaron condiciones socioeconómicas e higiénico-sanitarias más precarias que las mujeres atendidas por personal de los servicios de salud.

Mujeres con menor y mayor probabilidad de recibir atención del parto por personal de salud (público o privado). En el modelo logit construido ad hoc para la identificación de mujeres con menor y mayor probabilidad de recibir atención del parto por parte del personal de salud (cuadro V), el grupo de mujeres en "peor situación" quedó constituido por las que recibieron de 0 a 4 consultas durante la atención prenatal, con una escolaridad menor o igual al tercero de primaria y con un jefe de hogar dedicado a labores agrícolas (bondad de ajuste del modelo: devianza ${ }_{15}=20.212 ; p>0.05$ ). En este caso la probabilidad o proporción ajustada de recibir atención del parto por parte del personal de salud fue de 0.10 . El solo hecho de haber recibido cinco o más consultas de atención prenatal, aumentó esa probabilidad a 0.29 (2.9 veces más); haber tenido una escolaridad superior al tercero de primaria la aumentó de 0.10 a 0.31 , y el hecho de que el jefe del hogar se dedicase a labores no agropecuarias modificó la probabilidad de 0.10 a 0.26 .

Por otra parte, al comparar el grupo en "peor situación" con el grupo en "mejor situación" constituido por aquellas mujeres con un número de consultas de atención prenatal mayor a cinco, con escolaridad superior al tercero de primaria y con un jefe de hogar en labores no agropecuarias, la probabilidad observada pasó de 0.10 a 0.73 ; es decir, la suma de estas "condiciones favorables", en relación con la variable-respuesta, aumentó 7.4 veces la probabilidad de recibir atención del parto por personal de los servicios de salud (públicos o privados).

\section{Discusión}

Los resultados encontrados en la Región Fraylesca acerca de la atención del parto muestran condiciones notablemente más desfavorables que lo señalado por otras encuestas hechas en el ámbito nacional y en Chiapas en su conjunto. Mientras que en el país $85 \%{ }^{17}$ de los partos ocurridos entre 1989 y 1994, así como $89 \%$ de los habidos entre 1993 y $1995,{ }^{18}$ tuvieron lugar en alguna institución del sector público o privado, y alrededor de $12-13 \%{ }^{17,18}$ se dieron en casa de la mujer o de la partera durante esos periodos, en el área estudiada esta situación fue totalmente inversa: 7 de cada 10 partos se dieron en casa de la mujer o de la partera, y sólo uno de cada cuatro ocurrió en alguna institución pública.

De igual manera, la participación del personal médico en los partos dentro del área estudiada fue 2.3 veces menor a lo notificado por otras encuestas nacionales para el periodo $1993-1995^{18}(37 \%$ vs $86 \%$ ) y para $1994(89 \%) .{ }^{17}$ Asimismo, mientras que en el transcurso de 1995, en el ámbito nacional, 42\% de los partos fueron atendidos en hospitales públicos ${ }^{18}$ en el área estudiada este indicador fue 2.9 veces menor (14.5\%).

Prácticamente, en la región de estudio, dos de cada tres partos se dan fuera de las instituciones de salud (públicas o privadas), de los que casi 9 de cada 10 tienen lugar en casa de la mujer; a su vez, una gran proporción de estos últimos ocurren en condiciones higiénico-sanitarias sumamente inadecuadas y, en no pocas ocasiones, sin atención sanitaria alguna.

La baja proporción de partos atendidos por personal de las instituciones de salud en el área de estudio refleja una situación de evidente inequidad en el sistema de salud, particularmente en lo que se refiere a la utilización de servicios de salud. Los resultados

\section{Cuadro V

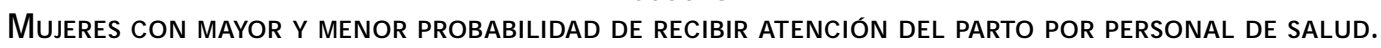 Region Fraylesca, Chiapas, Mexico, 1994}

\begin{tabular}{|c|c|c|c|c|c|}
\hline Condición de la mujer* & Momios & RM & $\mathrm{IC} 95 \%$ & Prob. de atención del parto ${ }^{\ddagger}$ & Razón de probabilidad \\
\hline Atención prenatal (2), jefe de hogar (2), escolaridad (2) & 2.796 & 18.11 & $7.14-45.91$ & 0.736 & 7.367 \\
\hline Atención prenatal (2) & 0.416 & 2.69 & $1.61-4.50$ & 0.294 & 2.935 \\
\hline Jefe de hogar (2) & 0.352 & 2.28 & $1.30-3.99$ & 0.260 & 2.602 \\
\hline Escolaridad (2) & 0.456 & 2.95 & $1.73-5.05$ & 0.313 & 3.132 \\
\hline $\begin{array}{l}\text { Atención prenatal (1), jefe de hogar (1), escolaridad (1) } \\
\text { * Atención prenatal: } 1=0-4 \text { consultas; } 2=5 \text { o más cons } \\
\text { Jefe de hogar: } 1=\text { labores agropecuarias; } 2=\text { labores nc } \\
\text { Escolaridad: } 1=\text { escolaridad de la mujer } \leq 30 \text {. de prim } \\
\text { ‡ Probabilidad de recibir atención del parto por person }\end{array}$ & $\begin{array}{l}\text { pecua } \\
=>3 \\
\text { salud }\end{array}$ & 1.00 & - & 0.100 & 1.00 \\
\hline
\end{tabular}


obtenidos muestran la existencia de un uso diferencial de los servicios de atención del parto, según condiciones socioeconómicas (indicadas por tipo de ocupación, escolaridad, ruralidad, condiciones de la vivienda, etc.; a mejor estatus socioeconómico, mayor utilización de los servicios institucionales y viceversa) y demográficas, y según uso y acceso de servicios de atención prenatal (número de consultas y tipo de agente que brindó la atención).

Este patrón diferencial en el uso de los servicios de atención del parto podría explicarse, según los hallazgos observados, entre otros factores, por: a) la presencia de barreras económicas y geográficas, así como culturales $^{4,19}$ (estas últimas no analizadas en el presente estudio), que impiden la utilización de los servicios abiertos por parte de la población más necesitada; b) inadecuados niveles de atención prenatal, y c) una percepción desfavorable de la calidad de los servicios ofrecidos en las unidades de salud institucionales.

Así fue posible observar, mediante el uso de modelos logit, que aspectos como la ocupación del jefe del hogar, el nivel de escolaridad de la mujer y la atención prenatal recibida (medida en número de consultas) permitieron identificar grupos de mujeres con mayor o menor probabilidad de recibir atención del parto por personal de salud; es decir, a medida que la situación de la mujer era "mejor", había una mayor probabilidad de que recibiera atención, y cuando la mujer se encontraba en "peor situación", la probabilidad era menor.

Ante tal panorama, es necesario mejorar la cobertura, la aceptabilidad y la calidad de los servicios institucionales de atención del parto. Para lo primero es preciso aumentar su accesibilidad, mediante la eliminación de barreras económicas y geográficas que limitan su uso. Esto se lograría mediante el fortalecimiento de las actividades extramuros que el personal de salud realiza en las comunidades que conforman el universo de trabajo. Una estrategia efectiva y de bajo costo sería la atención del parto mediante unidades móviles, las cuales constituyen una alternativa viable para disminuir los costos sociales de los servicios y estimular su uso. A su vez, las unidades móviles se apoyarían en un sistema de vigilancia epidemiológica para la detección de embarazadas de alto riesgo.

Por lo que respecta a la aceptabilidad de la atención del parto en establecimientos de salud, ésta se podría incrementar por medio de: a) la promoción de la atención del parto mediante la capacitación y el otorgamiento de apoyos al personal comunitario de salud (por ejemplo, a las propias parteras tradicionales, los "visitadores" y / o promotores de salud, etc.), y b) la creación de albergues o sitios anexos a las unidades de sa- lud en áreas rurales, donde las familias de las mujeres pudiesen alojarse, lo cual daría un ambiente más familiar y natural al proceso del parto y, al mismo tiempo, bajaría los costos sociales.

Respecto a la calidad de los servicios, ésta no podrá mejorarse sin: a) un programa de capacitación, supervisión y evaluación continua del personal adscrito a las unidades rurales de salud, complementado con un programa de estímulos e incentivos económicos que motive su superación y propicie el otorgamiento de servicios de mejor calidad, $\mathrm{y} b$ ) proveer a las unidades de salud de los recursos y equipos necesarios para la atención adecuada de partos no complicados. Para la atención de los partos complicados, se requiere fortalecer el sistema de referencia de pacientes al segundo nivel de atención.

Finalmente, el modelo propuesto para la identificación de grupos de mujeres con menor o mayor probabilidad de recibir atención del parto por personal de salud, cuyos resultados fueron consistentes con lo hallado para la atención prenatal en esta misma población, ${ }^{10}$ bien pudiera constituirse en una herramienta que permitiese orientar la priorización de esfuerzos y recursos, con el fin de mejorar las condiciones en que se desarrollan los partos de las mujeres identificadas como el grupo de "peor situación".

\section{Agradecimientos}

Los autores desean agradecer a María Luisa Estudillo y Roberto Solís, de El Colegio de la Frontera Sur, así como a María del Mar García Gil y David Prat, de la Universidad Autónoma de Barcelona, su invaluable participación en el trabajo de campo y en el análisis de resultados para este estudio.

\section{Referencias}

1. Fondo de las N aciones U nidas para la Infancia. El progreso de las naciones, 1996. Barcelona: UNICEF, 1996.

2. Gertler P, Rahman O, Feifer C, Ashley D. Determinants of pregnancy outcomes and targeting of maternal health services in Jamaica. Soc Sci Med 1993;37(2):199-211.

3.Valdés T, Gomáriz E, coord. Mujeres latinoamericanas en cifras. México, D.F.: Instituto de la Mujer/Ministerio de A suntos Sociales de España/Facultad Latinoamericana de Ciencias Sociales (FLAC SO ), 1993.

4. Castañeda X, García BC, Romero GX, N úñez-U rquiza R, González $H D$, Langer A. Traditional birth attendants in Mexico: Advantages and inadequacies of care for normal deliveries. Soc Sci Med 1996;43(2): 199-207.

5. Gobierno del Estado de Chiapas/C O PLADE. Plan Estatal de D esarrollo 1995-2000, Chiapas. 2a. edición. Tuxtla Gutiérrez: Gobierno del Estado, 1996:39-44 
6. Comisión N acional de Acción en Favor de la Infancia. México, Programa Nacional de Acción en Favor de la Infancia 1995-2000. Evaluación 1996. México, D.F.: Secretaría Técnica de la CN AFI (Serie Documentos Técnicos, núm. 2), 1997.

7. Secretaría de Salud. Estadísticas vitales 1992. México, D.F.: SSA, 1992. 8. García C, Flores M, Tovar V. México: comportamiento reproductivo y marginación social 1970-1990. Elementos para un diagnóstico geográfico en salud reproductiva. Salud Publica Mex 1995;37:279-287.

9. Potter JE. Utilización de los servicios de salud materna en el área rural. Salud Publica Mex 1988;30:387-402.

10. Sánchez-Pérez HJ, 0 choa Díaz H, García Gil MM, Martín-Mateo M. Bienestar social y servicios de salud en la Región Fraylesca de Chiapas: el uso de los servicios de atención prenatal. Salud Publica Mex 1997;39: 530-538.

11. Instituto N acional de Estadística, Geografía e Informática. XI Censo General de Población y Vivienda 1990. México, D.F.: IN EGI, 1990.

12. Consejo $\mathrm{N}$ acional de Población yVivienda. Indicadores socioeconómicos e índice de marginación municipal, México, 1990. México, D.F.: CO NAPO, 1993.
13. A gresti A. Categorical data analysis. N ueva York: John W iley and Sons, 1990.

14. U pton GJ.The analysis of cross-tabulated data. N uevaYork: John W iley and Sons, 1978.

15. Secretaría de Salud. N orma 0 ficial Mexicana para la Atención de la Mujer durante el Embarazo, Parto y Puerperio y del Recién N acido 1995. Diario 0 ficial de la Federación 1995.

16. Francis B, Green M, Payne C, ed. GLIM 4. The statistical system for generalized linear interactive modelling. N uevaYork: 0 xford Science Publications, 1993.

17. Dirección General de Epidemiología. Encuesta N acional de Salud Materno-Infantil 1994. México, D.F.: SSA, 1994.

18. Encuesta N acional de Planificación Familiar 1995.México, D.F.: CON APO, 1995

19. García C, C astañeda X, Romero X, González D, Langer A. Percepción de factores de riesgo reproductivo durante el embarazo por parteras del área rural de Morelos, México. Salud Publica Mex 1993;35:74-83. 